\title{
LA INVESTIGACIÓN AL SERVICIO DE LA DOCENCIA EN LAS FACULTADES DE MEDICINA PARA EL DESARROLLO DEL APRENDIZAJE AUTÓNOMO Y FORMACIÓN DE PROFESIONALES REFLEXIVOS
}

\author{
"Sócrates: Puedo contarte una tradición de los antiguos, que conocían la verdad. \\ Si nosotros pudiésemos descubrirla por nosotros mismos, \\ ¿nos seguiríamos preocupando aún de lo \\ que los hombres hayan pensado antes que nosotros?"
}

(PLATON)

\begin{abstract}
JORGE ENRIQUE ECHEVERRI SARMIENTO ${ }^{1}$
${ }^{1}$ Especialista en Medicina Interna, Nefrología y Cuidados Intensivos. Especialista en docencia Universitaria. Maestría en economía de salud y el medicamento. Jefe del servicio de nefrología Hospital Militar Central. Coordinador Académico Posgrado de Nefrología, Facultad de medicina Universidad Militar Nueva Granada. Bogotá, Colombia.

Correspondencia: je.echeverri.s@gmail.com
\end{abstract}

Recibido: 28 Agosto 2014 Aceptado: 4 Mayo 2015

\begin{abstract}
Resumen
Este artículo da una primera aproximación al papel que puede jugar la investigación en medicina, en el proceso de aprendizaje, dentro del desarrollo de profesionales autorregulados y autónomos. Para ello se propone partir de una reflexión pedagógica de aspectos referentes a la situación actual de la educación en medicina, posteriormente analizar ciertos roles y propósitos de la investigación al interior de las Facultades de Medicina, su interacción con los medios docentes y finalmente evaluar las estrategias educativas actuales para generar aprendizaje autónomo y herramientas de metacognición. Dentro del análisis se integran los resultados de entrevistas a profundidad aplicadas a estudiantes y docentes de diferentes facultades de medicina, cuyo objetivo fue explorar los aspectos mencionados. La investigación permite que el estudiante aborde al conocimiento desde cuatro perspectivas: desarrollar habilidades de metacognición, pensamiento complejo, apartarse de la concepción pasiva-tradicional y aprender a aprender.
\end{abstract}

Palabras claves: aprendizaje, cognición, enseñanza, educación, medicina, investigación. 


\title{
RESEARCHING AT THE SERVICE OF TEACHING IN MEDICAL SCHOOLS FOR DEVELOPMENT OF SELF-DIRECTED LEARNING AND REFLECTIVE PRACTITIONERS
}

\begin{abstract}
This paper aims to make a first approach to the role that researching can play in medical learning process and in development of self-regulated and self-directed learning professionals. A reflection of pedagogical aspects concerning the current situation of education in medicine is proposed, then an analysis of certain roles and purposes of researching in medical schools and its interaction with the world of education is presented and finally current educational strategies to generate autonomous learning and metacognition is evaluated. To explore these aspects, the results of structured interviews applied to students and teachers, from different medical schools, are given during the analysis. Researching allows the student to access knowledge from four perspectives: developing of metacognition skills, complex thinking, getting away from a passive and traditional point of view, and finally learning to learn.
\end{abstract}

Keywords: learning, cognition, education, teaching, medicine, researching.

\section{PESQUISA AO SERVICIO DO ENSINO NAS FACULDADES DE MEDICINA PARA O DESENVOLVIMENTO DO APRENDIZAGEM AUTÓNOMO E TREINAMENTO DE PROFISSIONAL REFLEXIVO}

\begin{abstract}
Resumo
Este artigo da uma primeira aproximação ao papel que pode jogar a investigação na medicina, no processo de aprendizagem, dentro do desenvolvimento de professionais autorregulados $e$ autónomos. Para isso começa-se com uma reflexão pedagógica de aspectos referentes a situação atual da educação em medicina, depois analisar alguns roles y propósitos da investigação nas faculdades de medicina, a interação com os médios docentes e finalmente, avaliar as estratégias educativa atuais para gerar aprendizagem autónomo y ferramentas de metacognição. No análises integram-se resultados de entrevistas com profundidade aplicadas aos estudantes e docentes de algumas faculdades de medicina. O objetivo das entrevistas foi explorar os aspectos mencionados. A investigação permite que o estudante aborde o conhecimento desde quatro perspectivas: desenvolver habilidades de metacognição, pensamento complexo, sair do concepto passivotradicional, e aprender a aprender.
\end{abstract}

Palavras-chave: aprendizagem, cognição, ensino, educação, medicina, investigação. 


\section{Introducción}

A menudo, como docentes universitarios, notamos que nuestros alumnos tienen problemas durante el aprendizaje de las ciencias médicas, a su vez, nos percatamos que muchos de los docentes no hacemos un alto en el camino con estos estudiantes y colocamos las responsabilidades del aprendizaje sólo en los estudiantes, sin darnos cuenta si hemos proporcionado las herramientas suficientes para desarrollar dicho proceso de aprendizaje autónomo. ¿Cómo hacemos para desarrollar en nuestros estudiantes en medicina autonomía y aprendizaje significativo? A la vez, nuestros alumnos perciben que los docentes no enseñamos tanto como debemos enseñar, que muchos de sus vacíos cognitivos son producto de no haber contemplado dichos temas en las aulas o en las prácticas clínicas.

Es necesario desarrollar estrategias permanentes que faciliten en nuestros alumnos y nosotros los docentes una cultura dirigida al desarrollo de habilidades metacognitivas y dar las herramientas para que los integrantes del medio universitario en medicina puedan aprender a aprender. ¿Estamos conscientes de la complejidad que implica desarrollar tales ambientes? ¿qué estrategias hay en la actualidad para desarrollar aprendizaje significativo? ¿Será que la investigación, tan popular en nuestros ambientes académicos en medicina, puede ser parte de estas? ¿Será que los obstáculos que vive el medio nacional en investigación son una barrera para el desarrollo de habilidades de aprendizaje en el medio universitario en medicina?

Con el fin de aproximarnos al papel que está jugando la investigación en la formación médica, e indagar sobre la participación en los procesos de aprendizaje y desarrollo de profesionales reflexivos, autónomos y críticos, se diseñó un instrumento con un modelo de preguntas abiertas para realizar entrevistas estructuradas a profundidad y dirigidas a profesionales médicos, aplicándose a residentes y docentes de diversas Facultades de Medicina que hubiesen participado en procesos de investigación durante su formación médica.

\section{La educación en medicina desde la óptica de un docente universitario}

Las escuelas de medicina en Colombia nacen aproximadamente a finales del siglo XVIII con la llegada de José Celestino Mutis a nuestro país y la formación de las primeras facultades de medicina a principios del siglo XIX (1). La influencia en el pensamiento médico de la Escuela Francesa, ha marcado profundamente la formación académica de nuestras escuelas, de manera que tradicionalmente el aprendizaje en los saberes médicos se ha centrado claramente en la figura clásica del maestro experto y conocedor de la verdad. Entendida ésta como expresión indiscutible de lo real y en la cual los docentes médicos han mostrado un papel dominante en el proceso educativo. Esta relación dominante ha permitido que el estudiante viva dos momentos pasivos dentro de su formación, el primero cuando asiste a clases magistrales de formación de las ciencias básicas y el segundo cuando asiste a las prácticas clínicas en donde pasivamente recibe del docente clínico la "apropiada" manera de evaluar y diagnosticar al enfermo. El monismo metodológico y la explicación causal de los fenómenos por leyes universales inquebrantables han mantenido la relación dominante y la separación maestro-alumno.

La ciencias médicas se han considerado como un área de conocimiento objetivo, único y racional sin espacios a la interpretación. La metodología probatoria y el conocimiento inductivo han primado claramente en la educación médica, es así como en los últimos veinte años se ha posicionado la denominada medicina basada en la evidencia, en donde los estudios clínicos y la epidemiología han logrado supuestamente comprobar que el conocimiento es incontrovertible. En resumidas cuentas, se ha considerado como fuente de conocimiento: la verdad del experto junto con la verdad de la evidencia epidemiológica, y el estudiante, ha venido desempeñando un papel pasivo $y$ expectante en su relación con el saber médico.

Nuestros estudiantes, con la expectativa de aprender a hacer, han centrado su formación en el desarrollo de habilidades técnicas para los procesos de diagnóstico y tratamiento de enfermedades, recibiendo como retribución el elogio del docente si demuestran saber lo que el experto quiere que sepa, es decir el alumno repite la doctrina que el maestro trasmite. El proceso de aprendizaje por imitación, basado en la observación del acto médico de sus docentes, es una característica homogénea de las diferentes escuelas. Siendo así que estas han logrado impregnar un estilo propio en sus alumnos, centrando su desarrollo en el saber individual, aprendizaje rígido y poco adaptable a los cambios de contexto. Esta doctrina ha permitido mantener un orden y control dentro del proceso formativo de una disciplina como lo es la medicina. A pesar de las razones históricas y la validez relativa que se pudiera considerar de estos estilos de aprendizaje, las necesidades actuales de desarrollo se ven limitadas en estos ambientes.

Uno de los cuestionamientos de nuestros procesos educativos en medicina son los riesgos inherentes a este esquema, producto de los cambios dinámicos que viene enfrentando nuestra cultura, tecnología y sociedad. La pérdida de la motivación estudiantil para profundización en 
el conocimiento, el proceso de aprendizaje frío no participativo, la confusión ante la variación de los contextos, restricción de la capacidad analítica, dilemas bioéticos, las necesidades de revertir el conocimiento hacia la sociedad como un motor de trasformación social, y la necesidad de la formación profesional ligada a la generación colectiva del conocimiento, ha obligado a replantear el ejercicio docente médico y generar propuestas diferentes en la educación para los futuros médicos.

En nuestro entorno, hemos concebido a la docencia como un proceso unidireccional, en donde el maestro transfiere sus conocimientos a sus estudiantes, teniendo la falsa impresión que estos logran integrarse instantáneamente. Sin embargo, para el proceso de generación de conocimiento es indispensable la interacción, es decir, una relación bidireccional entre el sujeto y el objeto, el aprendizaje significativo toma forma cuando el estudiante logra relacionar ese nuevo conocimiento con los previos, generando nuevos conceptos (2). No se desconoce el valor agregado de la teoría dentro del aprendizaje, pero ésta no puede ser entendida fuera de contexto, sin transformación, ni crítica. Una de las propuestas modernas de educación participativa es el aprendizaje basado en problemas, en donde se generan espacios apropiados para discutir, en grupos pequeños, temas médicos desde una óptica amplia y no fragmentada. Es así como se fomenta la discusión colectiva y se fortalece el trabajo en grupo (3).

Nuestras prácticas educativas deben ir más allá de la formación basada en análisis de casos clínicos aislados y nuestros maestros deben participar activamente en el acompañamiento de la construcción mutua de conceptos. Las clases magistrales deben ir transformándose en espacios de discusión colectiva para el fortalecimiento de la interpretación y la generación mutua del saber. Debe incorporarse la reflexión para darle sentido a los contenidos de nuestro saber, en nuestros estudiantes y futuros pares, a través de sus propias experiencias de aprendizaje. Se requiere renovar el desarrollo no sólo del conocimiento de las cogniciones sino también el conocimiento autorregulador, como habilidades metacognitivas indispensables para el desarrollo continuo del individuo.

\section{La investigación en la educación médica en Colombia}

Como lo refiere Otero (4), la investigación en medicina inicia en Colombia en el ambiente universitario, con la instauración de la cátedra de enfermedades tropicales a principios del siglo XX en la Universidad Nacional. Una década después el ministerio de salud crea una institución con funciones de gestión en temas en investigación en salud como es el Instituto Nacional de Salud. Desde ese momento las políticas del estado y el fuerte impulso de las facultades tradicionales de medicina, han venido desarrollando los grupos de investigación médica con el fin de mejorar la atención en salud y la calidad de medicina que se le ofrece a la sociedad. Esto llevó a que la investigación médica colombiana desde la década de los setenta estuviera orientada a la aplicación de tecnologías y conceptos generados en los países de primer mundo (4). Este tipo de investigación propiciaba en las facultades de medicina el desarrollo de protocolos estructurados y desarrollo de investigación formativa orientada a despertar la mentalidad investigativa.

La creación de Colciencias y su participación en este medio como financiador de la investigación ha facilitado la proliferación de unidades de investigación en universidades públicas y privadas con el fin de generar nuevos conocimientos. La conformación de grupos de investigación más estructurados, con fines puntuales de generación de un producto innovador y alta competitividad, ha venido desplazando la investigación formativa $y$ posicionando la investigación propiamente dicha (4). En el siglo XXI han aumentado los recursos nacionales para investigación, siendo involucradas permanentemente las universidades para la participación en ésta, diversificando las temáticas y dando una orientación social a la investigación, favoreciendo las temáticas de interés público como son las enfermedades crónicas, enfermedades infecciosas emergentes, lepra y tuberculosis, infecciones nosocomiales y enfermedades de trasmisión sexual como es el VIH (5).

La importancia y el rol que tiene la investigación en la universidad es un tema de amplia discusión académica en las últimas décadas. El tema de la investigación ha sido abordado desde diferentes ópticas, investigación formativa vs investigación propiamente dicha, generando un amplio debate en muchas áreas del conocimiento y en especial en las ciencias de la salud. Hoy en día la investigación es parte fundamental del quehacer universitario, se considera que su relación con la enseñanza es positiva y facilita procesos docentes, de allí que constituya uno de los tres pilares con la misión docente y de extensión. Desde la óptica de la enseñanza, la participación en investigación mejora las competencias del docente, desarrolla la infraestructura que se requiere para ella y mantiene actualizado un currículo. Es decir la universidad como fuente de conocimiento utiliza a la investigación como herramienta para el acceso a éste (6).

Al realizar las entrevistas a docentes y estudiantes se planteó como objetivo principal conocer en detalle lo que pensaban los entrevistados acerca de la investigación durante su proceso de formación médica, conocer cómo 
fueron sus primeras experiencias en investigación y cómo perciben ellos hoy en día que esas experiencias pudieron haber influenciado aspectos cognitivos, metacognitivos y de aprendizaje durante su formación médica. De igual forma el instrumento fue diseñado con el objetivo de explorar la importancia que tuvo la investigación para la formación médica de los participantes y estimar su impacto en los aspectos cognitivos y metacognitivos a través de escalas visuales análogas.

Se eligió una muestra por conveniencia de 15 médicos, todos ellos actualmente en instituciones universitarias, de los cuales 6 pertenecían a la planta docente de las instituciones y 9 médicos se encontraban cursando algún nivel de formación en especialidades médicas. (Tabla 1). Se realizaron entrevistas a profundidad, con grabaciones de aproximadamente 400 minutos, para posterior análisis por parte del investigador. Durante el registro digital y para facilitar el posterior análisis de la información se agruparon las respuestas acorde a unas áreas de exploración: experiencias en investigación, impresiones de pertinencia y utilidad de la investigación en las facultades de medicina, influencia de la investigación en la formación médica y aprendizaje, influencia de la investigación en el conocimiento de las cogniciones y en el conocimiento autorregulador; adicionalmente se analizaron las fortalezas y debilidades comunes encontradas entre los entrevistados en cada una de estas áreas de exploración.

Los resultados de las escalas visuales análogas fueron agrupados por quintiles para posteriormente poder ponderar resultados del grupo evaluado.

Tabla 1. Población entrevistada

\begin{tabular}{|c|c|c|c|c|c|c|}
\hline in & Sexo & Rol & $\begin{array}{l}\text { Nivel de } \\
\text { formación }\end{array}$ & $\begin{array}{l}\text { Escuela } \\
\text { Pregrado }\end{array}$ & $\begin{array}{l}\text { Primera } \\
\text { Especialidad }\end{array}$ & $\begin{array}{l}\text { Segunda } \\
\text { Especialidad }\end{array}$ \\
\hline 1 & $\mathrm{H}$ & Docente & Subespecialista & PUJ & PUJ & PUJ \\
\hline 2 & $\mathrm{H}$ & Docente & Subespecialista & PUJ & PUJ & PUJ \\
\hline 3 & $\mathrm{H}$ & Docente & Subespecialista & Bosque & Bosque & PUJ \\
\hline 4 & $\mathrm{M}$ & Docente & Subespecialista & JN Corpas & JN Corpas & JN Corpas \\
\hline 5 & $\mathrm{H}$ & Docente & Especialista & UMNG & UMNG & \\
\hline 6 & $\mathrm{M}$ & Docente & Especialista & Nacional & Nacional & \\
\hline 7 & M & Estudiante & Especialista & Nacional & Nacional & \\
\hline 8 & $\mathrm{M}$ & Estudiante & Especialista & Cauca & CES & \\
\hline 9 & $\mathrm{H}$ & Estudiante & Especialista & San Martín & UMNG & \\
\hline 10 & $\mathrm{H}$ & Estudiante & Especialista & Nacional & Bosque & \\
\hline 11 & $\mathrm{H}$ & Estudiante & Especialista & PUJ & UMNG & \\
\hline 12 & $\mathrm{M}$ & Estudiante & Especialista & Rosario & UMNG & \\
\hline 13 & $\mathrm{H}$ & Estudiante & Médico & UMNG & & \\
\hline 14 & $\mathrm{H}$ & Estudiante & Médico & UNAB & & \\
\hline 15 & $\mathrm{H}$ & Estudiante & Médico & Rosario & & \\
\hline \multicolumn{7}{|c|}{ UMNG } \\
\hline \multicolumn{7}{|c|}{ PUJ } \\
\hline \multicolumn{2}{|c|}{ Nacional } & \multicolumn{5}{|c|}{ Universidad Nacional (Bogotá) } \\
\hline \multicolumn{2}{|c|}{ Bosque } & \multicolumn{5}{|c|}{ Universidad El Bosque (Bogotá) } \\
\hline \multirow{2}{*}{\multicolumn{2}{|c|}{$\begin{array}{l}\text { Rosario } \\
\text { JN Corpas }\end{array}$}} & \multicolumn{5}{|c|}{ Universidad del Rosario (Bogotá) } \\
\hline & & \multicolumn{5}{|c|}{ Universidad Juan N. Corpas (Bogotá) } \\
\hline \multirow{2}{*}{\multicolumn{2}{|c|}{ San Martín }} & \multicolumn{5}{|c|}{ Fundación Universitaria San Martín (Bogotá) } \\
\hline & & \multicolumn{5}{|c|}{ Universidad CES (Medellin) } \\
\hline \multicolumn{2}{|c|}{ Cauca } & \multicolumn{5}{|c|}{ Universidad del Cauca (Popayán) } \\
\hline \multicolumn{2}{|c|}{ UNAB } & \multicolumn{5}{|c|}{ Universidad autónoma de Bucaramanga } \\
\hline
\end{tabular}

En general según los entrevistados, sus experiencias iniciales en investigación, no es en investigación propiamente dicha, sino en una primera aproximación a búsquedas de información y revisiones de tema. La participación en investigación durante la formación médica no es homogénea entre los entrevistados, observándose variabilidad entre las facultades de medicina, siendo un prerrequisito para el grado sólo en algunas de ellas. Los entrevistados que tuvieron un proyecto de investigación como una condición para graduarse, no desarrollaron proyectos adicionales al prerrequisito; por otra parte la mayoría de aquellos que participaron en investigación por motivación propia, participaron sólo en partes del proceso de investigación o en proyectos de líneas de investigación ya establecidas, sin llegar, en su mayoría, hasta la finalización de éstos.

Los entrevistados comparten que cuando se realiza algo novedoso la experiencia es muy positiva, la participación en investigación se considera muy satisfactoria por el hecho de encontrar cosas nuevas y entender mejor otras. La elección de la temática de investigación influye en la motivación posterior y la puesta en marcha del proyecto. Parece ser valioso para los entrevistados el hecho de aprender a generar dudas y luego preguntas, las cuales tomaban más sentido cuando se generaban en momentos de práctica. Las experiencias son más intensas en la medida que el estudiante materializa sus ideas, se involucra activamente en todo el proceso y se siente partícipe en la estructura de la pregunta, desarrollo, análisis y presentación de resultados. Cuando la participación en investigación genera un producto para publicación el estudiante adiciona mayor valor al trabajo realizado.

En pregrado, hay mayor inclinación por los aspectos metodológicos de la investigación, más en el hacer que en el desarrollar, mientras que las experiencias cobran importancia en el posgrado dado que el estudiante se involucra en todos los niveles de desarrollo de ésta y se comprende lo que se está investigando. Los entrevistados reconocen que la motivación en los proyectos de investigación se ve influenciada positivamente por el entendimiento metodológico y el conocimiento de los propósitos finales de esta.

Entre más claro y organizado fue el proceso de investigación más sentido y comentarios positivos alrededor del aprendizaje. La metodología juega un papel importante para no perderse durante el proceso de investigación, mantener la seriedad y el rigor del método. Adicionalmente, el desarrollo y curso de los proyectos de investigación parece ser poco predecible en la mayoría de los casos, sin embargo el grupo entrevistado comparte la necesidad de estructurar mejor las pautas y guías metodológicas para 
poder optimizar la inversión de tiempo y maximizar la experiencia. En términos metodológicos, las primeras experiencias fueron calificadas como valiosas, especialmente dado que mejoraban las habilidades para la búsqueda de la información, síntesis y registro de ellas en bases de datos.

Tabla 2. Resumen de las apreciaciones de los entrevistados.

\begin{tabular}{|l|l|}
\hline \multicolumn{1}{|c|}{ Experiencias negativas } & \multicolumn{1}{c|}{ Experiencias positivas } \\
\hline Falta de recursos & Docentes motivados \\
\hline Ausencia de planeación & Acompañamiento cercano \\
\hline Falta de compromiso & Inversión de tiempo por docentes \\
\hline Fallas metodológicas & Planificación y organización \\
\hline Falta de autonomía & Utilidad en la estructura profesional \\
\hline Temáticas no útiles & \\
\hline Análisis sin acompañamiento & \\
\hline $\begin{array}{l}\text { Tiempo de investigación } \\
\text { no protegido }\end{array}$ & \\
\hline
\end{tabular}

Adicional al desconocimiento de las líneas de investigación activas en las facultades de medicina, se identifican barreras para que los estudiantes ingresen a grupos preestablecidos de líneas de investigación: "a los estudiantes no se les ocurre, otro a los profesores no se les ocurre, muchos profesores no se sienten cómodos con la parte de la metodología de investigación, entonces, no se sienten cómodos trayendo gente a su estudio y prefieren no involucrar a alguien que venga a preguntar o cuestionar" (entrevistas).

La evaluación de la investigación en las facultades de medicina, según los entrevistados, en términos de pertinencia y coherencia es positiva, da sentido a la práctica diaria en medicina. Se resaltan los aspectos de la investigación aplicada y contextualizada (investigación en problemas esenciales), sin embargo, a pesar del valor que puede tener la investigación ajustada a las necesidades de la población y las dudas locales, los entrevistados coinciden en la necesidad de generar investigación para desarrollo científico y avance en áreas del conocimiento que permita dar valor internacional a éstas. Un punto en común es la necesidad de dar trascendencia a los productos de investigación universitaria, mejorar su alcance, más que ser un requisito de grado y poder tener continuidad en los hallazgos encontrados más allá del estudiante y el docente. En realidad se requiere más inversión institucional para darle coherencia a las líneas de investigación, poder profundizar en los vacíos del conocimiento y generar estructuras más fuertes de investigación que permitan llegar a los procesos finales de socialización.

En términos generales el grupo de entrevistados ubicó a la investigación en el quintil más alto de importancia con respecto a su influencia en la formación médica. El desarrollo de un pensamiento divergente, la flexibilización del pensamiento, la adquisición de la capacidad de cuestionar, criticar, ver los puntos de vista del otro, el estimular la curiosidad, el entender el modelo del método científico para solucionar problemas del quehacer diario, el conocimiento de cómo se profundiza en un tema, el conocer cómo buscar alternativas y poder mantenerse actualizado, el confrontar las conductas propias con lo que se encuentra en investigación, el entender los contextos y ubicar la validez de la información en ellos, el desarrollo de disciplina y una estructura metódica, el estímulo a la responsabilidad y el juicio ético son aspectos importantes identificados para la formación médica y desarrollados por la investigación.

Los currículos estrechos de la formación médica y el corto tiempo docente durante la carrera son limitantes para la continuidad de la investigación en los programa de formación, siguiendo un modelo educativo basado en la transmisión de conocimientos según los contenidos programáticos. La aproximación más común a investigación en los pregrados de medicina es a través de la cátedra de esta materia y la enseñanza de la medicina basada en evidencia o epidemiología clínica. Las líneas de profundización en grupos de investigación les permite a las universidades contar con un espacio para que los estudiantes se involucren. Sin embargo, el desarrollo de estos proyectos no necesariamente depende de los aportes de los estudiantes de pregrado y postgrado, convirtiéndose en ocasiones en apoyo técnico y no cognitivo. En efecto los intereses principales en estas líneas de profundización no siempre están orientados a la docencia sino al posicionamiento de los grupos de investigación en las instituciones (7).

Conceptos como los que comenta el Dr. Roselli (8), aún mantienen vigencia luego de 15 años de haber sido publicados:

Con algunas excepciones, que destacan más a individuos que a instituciones, la producción intelectual rara vez alcanza la calidad que se requiere según las normas internacionales, e incluso muy pocas de esas investigaciones válidas trascienden los límites de las publicaciones nacionales. A esto se agrega que son la excepción, y no la regla, las universidades que proporcionan incentivos para la producción intelectual de sus profesores y estudiantes....Aunque en años recientes se han visto esfuerzos de las universidades colombianas y de Colciencias por estimular la investigación y la producción intelectual, éstos difícilmente cerrarán la brecha que separa a Colombia no ya del mundo desarrollado, sino incluso de los vecinos de América Latina (8).

Con respecto al interés de los estudiantes de medicina por la investigación se puede encontrar diversos resultados entre 
las escuelas, especialmente relacionado con las barreras para desarrollar temas de investigación biomédica, los escasos recursos económicos designados, la carencia de conocimientos básicos y entrenamiento en metodología de la investigación, sumado a la incertidumbre por culminar los procesos, o ser exitosos en alcanzar resultados en las investigaciones (9). Factores como son el énfasis en las instituciones por la formación médica de profesionales para actividades netamente asistenciales, la competitividad y demanda académica propia de la carrera, la pobre remuneración de los investigadores en comparación con la práctica privada, mentores de investigación sin formación para la docencia, docentes catedráticos sin formación para la investigación y carencia de tiempo protegido son algunos de los aspectos adicionales que limitan la incorporación de los estudiantes de pregrado en investigación $(10,11)$.

La investigación es reconocida habitualmente como un aspecto importante de realizar en el proceso de formación médica, como lo muestra el estudio de Ángel y colaboradores (12), sin embargo, sólo un quinto de los estudiantes de pregrado en medicina deciden participar en investigación y menos del 10\% hacen parte formal de un grupo de investigación. Un tercio de los estudiantes refieren no investigar por ausencia de tiempo para hacerlo y un cuarto de ellos no sabe cómo hacerlo. Además del tiempo, la falta de asesoría y la poca flexibilidad constituyen las dificultades principalmente percibidas para hacer investigación. Cerca de la mitad de los estudiantes no tienen un modelo de investigador a seguir, mientras que el 70\% de los que investigan identifican a un docente a seguir, especialmente un médico investigador. Solo el 13\% reporta un trabajo de investigación terminado, siendo la minoría (2 al 3\%) quienes han logrado presentar los resultados del trabajo en un congreso o han realizado algún tipo de publicación. La mayoría de los estudiantes no sabe los pasos a seguir para publicar un trabajo en una revista científica (12).

A pesar de los esfuerzos de diferentes estudiantes en Colombia y el apoyo que algunas universidades les brindan a sus estudiantes para investigar, el número de estudiantes investigadores es bajo y las asociaciones científicas de estudiantes de medicina con frecuencia enfrentan problemas por escases de estudiantes dispuestos a investigar (12).

La motivación en los estudiantes puede ser la fuente motora más importante que los mantiene en la decisión de involucrarse en investigación por lo cual es crucial promoverla en las etapas tempranas de la formación médica. En efecto, el desarrollo de habilidades en la búsqueda y aproximación a la literatura médica, su importancia para el desempeño en futuras especialidades, la posibilidad de generar publicaciones y mejorar el currículo vitae, los vínculos y contactos generados en investigación y la experiencia de campo constituyen otras consideraciones importantes que inclinan a los estudiantes a participar en investigación.

\section{Modelos educativos en medicina orientados a favorecer el aprendizaje autónomo y significativo}

La educación en medicina ha tratado de explorar nuevas estrategias de enseñanza y de formación con el fin de superar las barreras de los modelos tradicionales transmisionistas enfocados en contenidos estáticos, fragmentados y no ajustados a los retos de la modernidad que obligan a la transformación permanente. Un nuevo enfoque de enseñanza es el denominado aprendizaje basado en problemas (ABP), el cual nace en 1969 en la Facultad de Medicina de la Universidad de McMaster (13).

El ABP es una estrategia inscrita en modelos pedagógicos activos de aprendizaje por descubrimiento que rompe con los esquemas tradicionales en la enseñanza de la medicina, favorece el aprendizaje significativo mediante una visión integral del objeto de estudio y sitúa al estudiante en el contexto de su propia realidad, permitiéndole el desarrollo de competencias analíticas, argumentativas, propositivas, sociales e interpersonales con base en la búsqueda planificada de la información para la solución de problemas (14).

Las experiencias más activas del estudiante en su aprendizaje, el énfasis en su autonomía, el desarrollo de pensamiento crítico y su objetivo centrado en el tránsito del estudiante por caminos similares a los que la ciencia recorrió para encontrar conclusiones hacen de este método una estrategia pedagógica atractiva. De allí, que la utilización de esta metodología se haya difundido por el mundo, como lo demuestra Finucane y colaboradores en donde en Australia para finales del siglo pasado, más de las mitad de los médicos australianos graduados provenían de programas ABP y muestran cómo esta estrategia estimuló e hizo grato el aprendizaje, desarrollando interés y autoaprendizaje (15).

El ABP se fundamenta en iniciar el proceso educativo en el planteamiento de una situación problémica, permitiendo que el estudiante identifique sus necesidades para poder analizar y comprender integralmente esta situación, proponiendo unos desarrollos temáticos para poder proponer soluciones, generando un pensamiento crítico e integrando conocimientos previos con los adquiridos en el momento (16).

El desarrollo del conocimiento en el estudiante termina 
siendo producto de su construcción y la fundamentación ajustada a su contexto, es así como el ABP podría compartir elementos propios de las teorías del constructivismo de Piaget y Vigotsky (2).

Adicionalmente los vínculos sustantivos y no arbitrarios entre el conocimiento previo y el nuevo permiten dar significado al aprendizaje como lo menciona Ausubel. El aprendizaje significativo implica claramente la reevaluación y transformación de éste, la interconexión con los presaberes y el uso o aplicación en los diferentes contextos (17).

El ABP no constituye solamente una estrategia de aprendizaje, se fundamenta en el aprendizaje dentro de un contexto, facilitando la conexión de los conceptos con los ambientes y la realidad del estudiante, inicia con la activación del conocimiento predecesor del estudiante al analizar una situación y definir sus necesidades de profundización y ampliación temática, el denominado conflicto cognitivo que estimula el aprendizaje y la posterior construcción de los propios referentes ligados a su entendimiento y construcción de conocimiento (16).

El estudiante en el ABP es el centro del proceso y se convierte en el gestor del conocimiento, fortaleciendo la interacción con otros integrantes del grupo gracias a actividades pre-establecidas de procesos de retroalimentación colectiva. Los ambientes generados en el ABP permiten generar espacios de confianza mutua, debate académico basado en el respeto y escucha del otro, evaluando las construcciones de los entendimientos individuales y generando espacios de negociación social, basados en la argumentación, con fortalecimiento de las respectivas habilidades de comunicación (13).

Algunos de los aspectos importantes de mencionar para el adecuado ejercicio de los problemas propuestos en el ABP encontramos: la situación debe captar y comprometer el interés del alumno, ésta debe relacionarse con conocimientos previamente adquiridos, los problemas deben estar orientados a la toma de decisiones y a generar juicios basados en la integración de varias fuentes de información, se debe estimular la cooperación entre todos los integrantes del grupo y espacios de dialogo, no es indispensable que exista una sola solución para este, especialmente si se integra un componente propio de opinión, los docentes deben estar enfocados en facilitar y estimular el proceso más que dirigirlo (14).

Los aspectos mencionados ponen en concordancia al ABP con el marco ideológico del constructivismo, en donde los conocimientos deben ir de la mano del desarrollo de habilidades especialmente orientadas al desarrollo de autonomía, jerarquización, identificación y síntesis de lo relevante, honestidad intelectual, diseño de propuestas auténticas, análisis crítico, pensamiento complejo integrador contextualizado e interdisciplinario, ofreciendo espacios de reflexión, fomentando espacios de duda sistemática y de aplicación del conocimiento.

\section{Conceptos generales de metacognición y su participación en el desarrollo de individuos autónomos y reflexivos}

La metacognición es un área de reciente estudio derivada del interés por encontrar estrategias eficientes para la enseñanza de autonomía en el aprendizaje, logrando identificar las bases de las distinta operaciones mentales con el fin de saber cómo, cuándo y para qué debemos usarlas. Como refiere Buron que decía Flavell (1978), un especialista en psicología cognitiva infantil y pionero del estudio de la metacognición, "la metacognición hace referencia al conocimiento de los propios procesos cognitivos, de los resultados de esos procesos y de cualquier aspecto que se relacione con ellos; es decir, el aprendizaje de las propiedades relevantes de la información y de los datos" (18).

Si bien la palabra metacognición significaría "más allá del conocimiento", quizás es mejor llamarla "conocimiento autoreflexivo" dado que se refiere al conocer del cognoscente, dando más énfasis a las funciones autoreguladoras y no solo las cognitivas. Es así como la metacognición nos permite ser conscientes de los procesos de aprendizaje y poder intervenir en ellos (19). Su primer componente permite el control de los procesos cognitivos y el segundo se relaciona con las experiencias metacognitivas que permiten generar estrategias de planeación y gestión de dichos procesos cognitivos (20).

La metacognición aborda tanto el conocimiento individual de las cogniciones, dentro de las cuales encontramos operaciones mentales (escritura, lectura, memoria, atención, percepción, comprensión, planificación, comunicación, creatividad, motivación), como también el conocimiento las funciones autoreguladoras de las actividades mentales (reflexión, interpretación, determinación, autoevaluación, autonomía, crítica); estas últimas se proponen como funciones integradoras del comportamiento. Si quisiéramos diferenciar entre las habilidades cognitivas y metacognitivas pudiéramos decir como Rivers (21) que las primeras las utilizamos para ejecutar una tarea (saber hacer), mientras las ultimas nos ayudan a entender como se ejecutó (conceptualizaciónabstracción).

Tanto para el conocimiento de las cogniciones como para el 
conocimiento autorregulador, los entrevistados consideran que la investigación facilita ambos procesos en cada uno de sus contenidos (Tabla 3 y 4), existiendo mayor énfasis por las primeras (Fig. 1 y 2.). Se resaltan aspectos como la planificación, comprensión, lectura y escritura dentro de las cogniciones, mientras que la crítica, interpretación y reflexión se destacan en las autoreguladoras.

Tabla 3. Influencia de la investigación en el conocimiento de las cogniciones

\begin{tabular}{|l|c|}
\hline Cognición & Quintil de importancia (Media) \\
\hline Memoria & 3 \\
\hline Escritura & 4 \\
\hline Lectura & 4 \\
\hline Atención & 4 \\
\hline Percepción & 4 \\
\hline Comprensión & 4 \\
\hline Planificación & 4 \\
\hline Comunicación & 4 \\
\hline Creatividad & 4 \\
\hline Motivación & 5 \\
\hline $\begin{array}{l}\text { 1er Quintil: Sin influencia por la participación en investigación } \\
\text { 5to Quintil: Altamente influenciado por la partición en } \\
\text { investigación }\end{array}$ \\
\hline
\end{tabular}

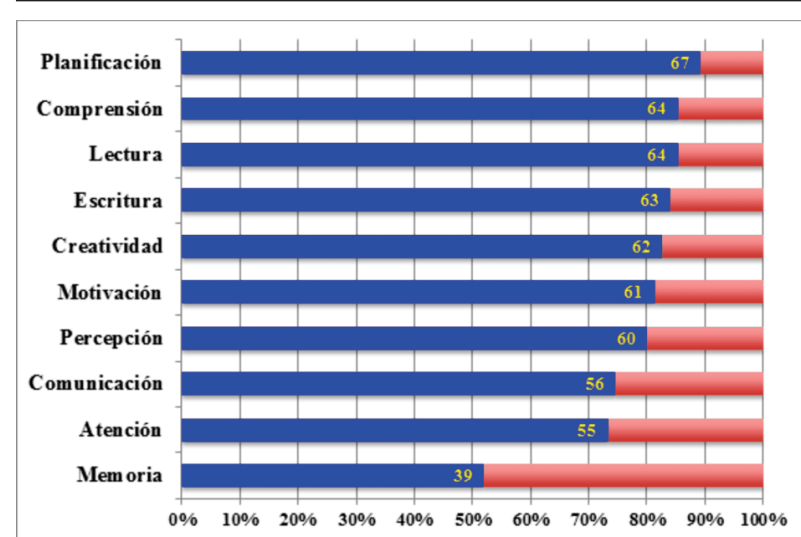

Figura 1. Influencia de la investigación en el conocimiento de las cogniciones

Tabla 4. Influencia de la investigación en el conocimiento autoregulador

\begin{tabular}{|l|c|}
\hline Reflexión & 2 \\
\hline Interpretación & 2 \\
\hline Determinación & 2 \\
\hline Autoevaluación & 2 \\
\hline Crítica & 2 \\
\hline Autonomía & 3 \\
\hline $\begin{array}{l}\text { 1er Quintil: Importancia Central. Muy influenciado por la } \\
\text { investigación } \\
\text { 5to Quintil: Importancia marginal. Pobre influencia } \\
\text { por participación en investigación }\end{array}$ \\
\hline
\end{tabular}

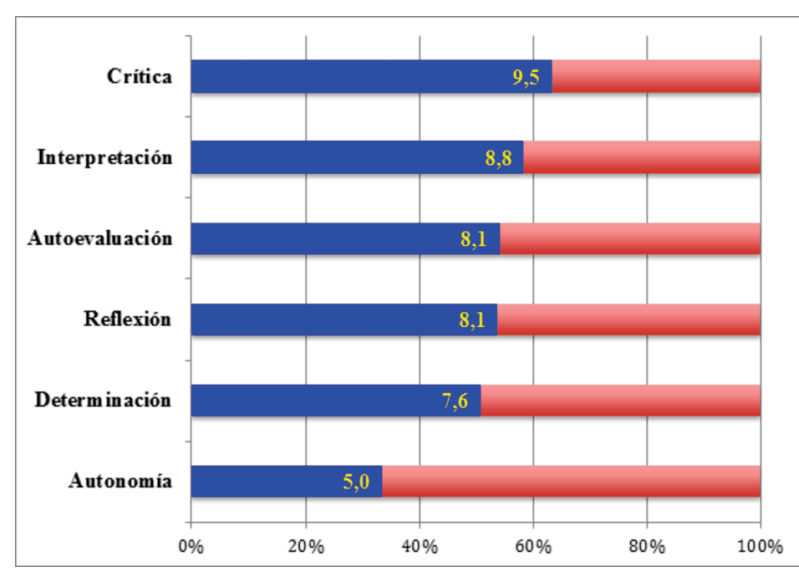

Figura 2. Influencia de la investigación en el conocimiento autoregulador

En una escala de 1 a 5, se encontró gran similitud al analizar la influencia de la investigación en el conocimiento de las cogniciones acorde a las perspectivas de los docentes y de los estudiantes entrevistados (Figura 3). En general los estudiantes dieron mayor valor al desarrollo de habilidades referentes a la escritura, lectura y atención, mientras que los docentes favorecieron a la creatividad, percepción y motivación.

Este mismo ejercicio se replicó en el análisis del conocimiento autorregulador (Figura 4), encontrando diferencias importantes. La interpretación y autoevaluación fue calificada por los estudiantes como aspectos mas influenciados por la investigación mientras que los docentes favorecieron a la crítica.

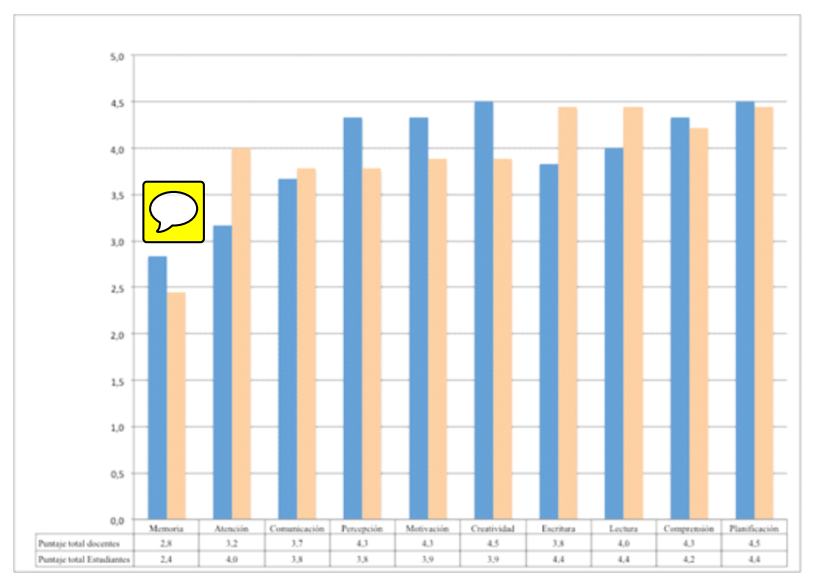

Figura 3. Influencia de la investigación en el conocimiento de las cogniciones. Perspectiva docente vs estudiante 


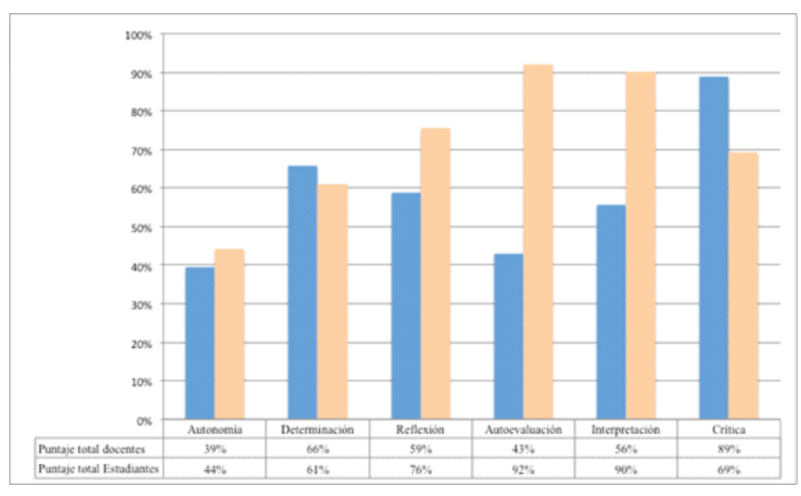

Figura 4. Influencia de la investigación en el conocimiento autoregulador. Perspectiva docente vs estudiante

Tabla 5. Aportes específicos al conocimiento de las cogniciones

\begin{tabular}{|c|c|}
\hline Áreas de mayor influencia & Áreas de menor influencia \\
\hline Lenguaje & Memoria \\
\hline $\begin{array}{l}\text { Desarrollo lenguaje científico } \\
\text { Aprender su uso } \\
\text { Desarrollo de síntesis } \\
\text { Objetividad de expresión } \\
\text { Claridad en la exposición }\end{array}$ & $\begin{array}{l}\text { Evocación y recuperación } \\
\text { de estudios relevantes }\end{array}$ \\
\hline Lectura & Atención \\
\hline $\begin{array}{l}\text { Hábito de lectura } \\
\text { Lectura crítica, analítica, comparativa } \\
\text { y relacional } \\
\text { Identificación de los vacíos del } \\
\text { conocimiento } \\
\text { Generación de preguntas }\end{array}$ & $\begin{array}{l}\text { Enfoque en lo relevante } \\
\text { Identificación de los detalles } \\
\text { Práctica diaria como fuente } \\
\text { de nuevas preguntas }\end{array}$ \\
\hline Comprensión & Comunicación \\
\hline $\begin{array}{l}\text { Profundización temática } \\
\text { Manejo de múltiples informaciones } \\
\text { Generar y aclarar dudas }\end{array}$ & $\begin{array}{l}\text { Canales interdisciplinares } \\
\text { Habilidades de expresión } \\
\text { Trasmisión eficiente } \\
\text { Trabajo en equipo } \\
\text { Síntesis de resultados }\end{array}$ \\
\hline \multicolumn{2}{|l|}{ Planificación } \\
\hline $\begin{array}{l}\text { Rigor metodológico y disciplina } \\
\text { Orden logístico, académico } \\
\text { Manejo de tiempo y recursos } \\
\text { Identificación de factores de cambio } \\
\text { Replanificación }\end{array}$ & \\
\hline \multicolumn{2}{|l|}{ Creatividad y motivación } \\
\hline $\begin{array}{l}\text { Motor inicial de la investigación } \\
\text { Fuente de desarrollo } \\
\text { Perfeccionamiento de preguntas } \\
\text { Fuente de innovación } \\
\text { Resolver dudas genera satisfacción }\end{array}$ & \\
\hline
\end{tabular}

\section{Aportes específicos al conocimiento autorregulador}

Se consideró a la reflexión y la crítica como dos aspectos fundamentales influenciados por la participación en investigación y asociados al pensamiento divergente. El enfrentarse con un fenómeno y tratar de buscar explicaciones, ver si éstas son apropiadas, si existen otros factores, la utilidad e impacto que pueden tener los hallazgos durante la investigación, son aspectos que favorecen el desarrollo de reflexión y la crítica. La investigación biomédica obliga a reflexionar sobre aspectos bioéticos, sobre las propias conductas, sobre la práctica diaria, sobre problemas inherentes al proceso de investigación y sobre cómo mejorar el entorno. La capacidad de análisis de la información y el pensamiento crítico se fortalece por la investigación y a medida que hay más participación en ella, como lo refieren los docentes entrevistados.

La investigación invita a ver resultados no sólo en términos de un número frío sino entender su significado, la influencia de los contextos, abstraer de la información lo relevante y extrapolable, es así como los estudiantes entrevistados consideraron éste como uno de los aspectos que más se desarrolla cuando se participa en investigación. Esta herramienta permite explicar mejor los fenómenos que analizamos y tener un pensamiento objetivo y estructurado de la realidad médica.

Con respecto a la determinación, hubo dos apreciaciones importantes que se logró identificar en las entrevistas. El primero de ellos está relacionado con el desarrollo de seguridad en la toma de decisiones en la práctica diaria, en el cual la práctica de investigación forma profesionales con mayor capacidad de tomar decisiones sobre conductas a seguir. "Mi práctica cambio desde que hago investigación, mientras otros dudan yo no, yo tengo certeza de la utilidad de algo" (entrevistas). El segundo de ellos hace relación al desarrollo de determinación durante los procesos de investigación con el fin de lograr finalizar los proyectos. Desde ambas apreciaciones nuestro medio de investigación obliga a los estudiantes a desarrollar estas habilidades.

La participación en investigación, especialmente en aquellos que logran estar durante todo el proceso, facilita el desarrollo de autonomía y autoevaluación, aspectos más asociados por los entrevistados con la madurez temática en medicina, la praxis y la proposición de nuevos proyectos. En general se considera que existen muchas limitantes en nuestro medio para el desarrollo de autonomía, aspectos tratados con anterioridad, mientras que los estudiantes consideran que la investigación los invita a realizar procesos de autoevaluación posiblemente asociado a su etapa de formación médica.

"La investigación es a la docencia como el pecado es a la confesión, si uno no hace nada de lo primero no tiene nada que decir de lo segundo, decía el fundador de la Universidad de Cornell". Los entrevistados coinciden que la investigación puede facilitar los ambientes de enseñanza y a la vez estimular los procesos de aprendizaje. La 
investigación facilita la organización estructural del estudiante para poder enfrentar mejor las temáticas, mejorar la comprensión de aspectos teóricos y prácticos del conocimiento médico, desarrolla habilidades propias del procesamiento de la información, ayuda a descubrir como se obtiene el conocimiento de la naturaleza y aporta herramientas de juicio necesarias para poder ser objetivos, al final nos enseña como podemos lograr aprender más de un tema, el tema que estamos investigando, permitiendo poder replicar esta experiencia bajo un método sistemático.

Autores como Buron (18) consideran un individuo metacognitivamente maduro cuando logra saber qué quiere conseguir (conocimiento declarativo), cómo conseguirlo con las estrategias adecuadas (conocimiento procedimental), cuándo incorporarlo a la toma decisiones (conocimiento condicional), cómo controlarlo, evaluarlo y regularlo acorde a los contextos.

"El desarrollo metacognitivo es motivacional por naturaleza: un alumno metacognitivamente desarrollado generalmente conoce el esfuerzo que requiere una tarea, posee recursos para realizarla, tiene conciencia de que el esfuerzo le lleva a un rendimiento superior y por consiguiente está motivado" (22).

Según refiere Otake (23) que decía Flavell, el conocimiento metacognitivo resulta de la interacción de la persona, la tarea y la estrategia. La persona entendida como la representación que tiene el individuo de sus capacidades, limitaciones y sus propios conocimientos. La tarea, como la representación que tiene el individuo sobre las condiciones propias de lo que debe realizar y su grado de dificultad. La estrategia, entendida como los conocimientos referentes a las diferentes formas a ejecutar según los diferentes procesos cognitivos.

"El objetivo no es que los alumnos conozcan qué estrategias deben usar, sino que lo más importante es que trabajen estratégicamente. Se trata, pues, de crear una situaciones de aprendizaje que les obliguen a usar las estrategias de forma continuada para que se conviertan en hábito de trabajo y estilo de aprendizaje." (18)

Se han propuesto diversas estrategias didácticas para desarrollar habilidades metacognitivas en los estudiantes, dentro de ellas encontramos el modelamiento metacognitivo, la discusión metacognitiva y la autointerrogación metacognitiva. El primero de ellos se fundamenta en generar espacios en donde se presenta a la planeación, el control de la ejecución y la evaluación como tres pasos importantes para la realización de una tarea, trabajando aspectos como la priorización de actividades, organización de procesos, verificación de resultados parciales, aseguramiento de procesos para el logro de resultados y reflexión final evaluando pros y contras en la ejecución de la tarea. La discusión metacognitiva permite tomar conciencia sobre los niveles de conocimiento y las estrategias individuales de aprendizaje, el intercambio con pares y docente permite el aprendizaje bidireccional. Por último, la autointerrogación metacognitiva está orientada a estimular en el estudiante durante todo el proceso la formulación de preguntas alrededor de la ejecución de la tarea (24)

En resumen se puede proponer a la metacognición como un método inteligente de aprender en el cual se obtiene un adecuado resultado, mayor rendimiento sin aumentar el esfuerzo, mayor eficacia. Esta estrategia utiliza la auto observación de los procesos implicados en el aprendizaje y el ejercicio de habilidades auto reguladoras para encontrar por si mismo la ruta más eficiente, fijándose más en los procesos que en los productos finales. Centra el proceso en el alumno y no en el docente, permitiendo el crecimiento no sólo cognitivo sino habilidades de autonomía, reflexión y crítica.

\section{Participación de la investigación en procesos de formación de médicos autónomos, críticos y reflexivos}

Los modelos educativos contemporáneos han planteado como base del aprendizaje la construcción permanente del conocimiento, siendo éstos adoptados particularmente en los espacios universitarios e incorporándose en sus propósitos básicos. Como bien refiere Vélez que decía Leonardo Da Vinci: "El que no cita más que autoridades demuestra hacer uso de su memoria pero no de su razón". Esta concepción estática del conocimiento ha impactado en los modelos educativos de forma que el proceso de aprendizaje tradicional toma al estudiante como un contenedor en donde se deposita información, muchas veces fuera de contexto y no integradas (25).

En la actualidad, con un incremento desproporcional de las fuentes de información, es necesario generar herramientas en los estudiantes orientados al análisis crítico de ésta, no sólo en cuanto a la validez y relevancia de la información sino también a la estrategia de construcción de conocimiento. No siempre es fácil generar responsabilidad y motivación para aprender, se requiere de organización, planificación, estrategias y recursos cognitivos para no perder la ruta y adicionalmente la preparación de nuestros estudiantes de medicina debe estar orientada a el desarrollo de habilidades comunicativas que permitan incorporar diversos saberes y la interdisciplinaridad. Los estudiantes deben obtener además de la perspectiva biológica, aspectos sistémicos como son el análisis del contexto social, 
psicológico, tecnológico y cultural.

La propuesta de investigación permite acercar a los estudiantes al conocimiento de manera vivencial, vinculando la teoría con la práctica y desarrollando habilidades de análisis e interpretación. Por otra parte permite que el docente refuerce su decisión por la búsqueda del conocimiento, flexibilizando su saber y permitiendo reafirmar el carácter dinámico que éste tiene. Es así como el docente e investigador puede contribuir al enriquecimiento de su microcurrículum a través de las líneas de profundización que se generan en la investigación, evitando los escenarios monótonos de la repetición (6).

"Convertir la información disponible en un conocimiento organizado y con sentido requiere la capacidad de reflexionar, de tener criterios claros y saber enfrentar la angustia que produce el encuentro con la incertidumbre de no saber respuestas exactas y tranquilizadoras frente a las preguntas que plantea la misma realidad" (24).

Desde la óptica del aprendizaje sabemos que este es más eficiente cuando el individuo tiene una experiencia de vida, cuando descubrimos por nosotros mismos algo que nos motiva y cuando ese conocimiento nos genera un acto de reflexión. La investigación permite que el estudiante pueda abordar al conocimiento desde estas tres perspectivas, desarrollar habilidades de metacognición, pensamiento complejo, apartarse de la concepción pasiva tradicional y al final aprender a aprender. La formación integral a través de la investigación se basa en la puesta en acción del conocimiento para solucionar situaciones complejas y dilemáticas. Sumado a esto se mantiene abierto el interés por la búsqueda de lo nuevo, la creatividad e innovación que puede tener un individuo en formación desarrollando confianza en la búsqueda del conocimiento (6).

De igual manera, es importante tener en cuenta que uno de los propósitos del aprendizaje más importantes consiste en establecer los vínculos y conexiones necesarios para que todos los conocimientos teóricos generados sean puestos en práctica y adicionalmente sea transmitido a las nuevas generaciones. El descubrimiento de conocimientos a través de la investigación permite al estudiante ubicarse en los contextos, aplicar las bases teóricas preexistentes y lograr identificar las particularidades de su medio que pueden impactar en el proceso de investigación e influenciar los resultados. La investigación acerca al estudiante a su realidad social, mostrándole los aspectos prácticos del conocimiento, su relación con los contextos y posiblemente logra relativizar ciertas "verdades" que pudieran ser absolutas en los textos. Anudado a esto, la investigación pretende que los docentes puedan de igual forma reflexionar sobre su realidad, permitiendo reconstruirla y ajustarla a su contexto, algo que es necesario en países como Colombia en donde el conocimiento es extrapolado esencialmente del generado en los países del hemisferio (25).

El conocimiento de las actividades mentales durante la investigación, como son las finalidades de la lectura, experimentar por qué es importante recordar, el juicio alrededor de lo que se encuentra, cómo regular lo que se tiene que escribir y qué no es importante, qué es precisión comunicativa, cuáles son los elementos importantes para tomar decisiones, en qué se diferencia comprender de memorizar, cómo la comprensión cambia nuestro actuar, qué es honestidad intelectual, cómo realizar preguntas y reconocer qué es lo que se sabe y qué no, cómo el reconocer las dudas lleva a la prudencia y motiva a la búsqueda de respuestas.

"El primer rasgo de la metacognición es precisamente la toma de conciencia de la eficacia de los propios procedimientos". Esta toma de conciencia no sólo debe empezar en los alumnos sino que es necesario que los profesores tengan una idea clara de lo que quieren cuando se asigna una labor a un estudiante, cómo debe trabajar el alumno para lograr esos objetivos, participar en la enseñanza de esos procesos óptimos para lograrlo y utilizar unos recursos apropiados para monitorizar ese camino (18). Parece ingenuo pensar que debemos seguir creyendo que la responsabilidad de encontrar los medios apropiados para aprender a aprender es responsabilidad neta del alumno. No podemos olvidar que a pesar que nuestras experiencias de enseñanza-aprendizaje pudieron ser buenas, eso no implica que no puedan ser mejoradas. Los estudiantes pueden tener la falsa idea que sus métodos de trabajo les han servido para mantener un rendimiento, pero es este aspecto el que puede hacer que en el futuro los conocimientos preexistentes puedan llevar a no tener flexibilidad en nuevos aprendizajes, de hecho cuando pensamos que todo se sabe se pierde la curiosidad por la continua búsqueda del conocimiento (18).

Cada cultura propone para el aprendizaje la formación de una "cultura de aprendizaje, una forma de relacionarse con el conocimiento, que está esencialmente mediada por los sistemas de representación en que ese conocimiento se conserva y se transmite, en suma, por las tecnologías de conocimiento dominantes en una sociedad" (26).

Debemos desprenderlos de los preceptos que nos impulsan a darle el peso transmisionista de los contenidos y generar espacios para poder afianzar herramientas que proporcionan madurez y autonomía en el aprendizaje, capacidad de tener pensamiento complejo, contextualizado y de trabajar por sí mismo, sabiendo lo esencial y logrando 
incorporarlo a su práctica, enfrentándose mejor a las exigencias cotidianas, prepararlo para la adquisición de nuevos conocimientos. "Se escribe sólo de aquello que se sabe; más aún, de aquello que se sabe y se comprende; $y$, mucho más todavía, sólo de aquello que se sabe, se comprende y se aprecia" (27).

No sólo basta con mostrar la utilidad de la investigación para el ejercicio profesional sino que los estudiantes participen activamente en ella para que vivencien por si mismos sus ventajas y posteriormente retroalimentar la experiencia. Se deben preferir espacios en donde el estudiante pueda trabajar por sí mismo y autorregular su sistema de trabajo, favoreciendo autonomía y autoreflexión. Para asegurar la transferencia del entrenamiento, que garantiza usar los conocimientos en contextos nuevos y diversos es necesario enseñar habilidades de autocontrol (28).

\section{Conclusiones}

El paradigma actual educativo está enfocado en el proceso de aprendizaje más que en la enseñanza y se centra en lo que está sucediendo en el cognocente más que en el ambiente (contenidos, currículo, recursos, universidad). Cada vez es más importante incentivar el análisis crítico contextualizado, de forma que se desarrolle en el profesional el carácter para poder tener criterio que le permita tomar decisiones frente a las situaciones que se le presenten en su práctica médica. Esto demanda que durante el proceso de enseñanza, se suministren herramientas de juicio que faciliten el establecimiento de su propio criterio. Adicionalmente, dado que no existe una sola forma de aprender, y los estilos de aprendizaje pueden variar acorde a los contextos, factores interno-externos y elementos propios de cada alumno, debemos crear espacios para la experiencia de aprendizaje autónomo e individualizado. Espacios que deben permitir al estudiante reconstruir su propia representación del mundo externo, basándose en la comprensión, interpretación, uso de la información y logrando autorregular su propia actividad. Espacios abiertos, que de igual forma, partiendo de la concepción flexible del conocimiento permitan y estimulen la discusión y el diálogo, el trabajo argumentativo en equipo, momentos de reflexión y de creación de conocimiento, como instrumento para la acción y que se modifica en la acción.

El apropiado desarrollo de la investigación en la academia, a pesar que la forma como se desarrolla actualmente adolece de múltiples debilidades o restricciones, puede constituir una herramienta útil para explorar sistemáticamente y facilitar cada uno de estos aspectos, desarrollar habilidades en los estudiantes enfocadas en el conocimiento de las cogniciones, el pensamiento complejo integrador, el conocimiento autoregulador y apartarse de la concepción pasiva tradicional. En la exploración bajo entrevistas a profundidad se encontró que existe una alta valoración de la investigación como método de desarrollo de habilidades cognitivas, exploración del conocimiento interdisciplinario, crítica, desarrollo de autonomía y entendimiento de los contextos.

El hacer los resultados de lo que sucede en la práctica, en nuestro caso la investigación, es una de las mejores formas de desarrollar criterios en los alumnos y docentes para mejorar el proceso de enseñanza de la medicina, el aprender a aprender del estudiante y asegurar una mejor práctica médica. Estos resultados nos invitan a profundizar en nuevos horizontes en investigación pedagógica: cuantificar la magnitud del impacto en la formación médica de los programas de investigación en las facultades de medicina.

\section{Conflicto de intereses}

El autor declara no tener de manera directa o indirecta, ningún tipo de conflicto de intereses financieros, académicos o laborales que puedan poner en peligro la validez de este estudio.

\section{Financiación}

Este trabajo no requirió apoyo financiero.

\section{Agradecimientos}

El autor quiere agradecer a todos los que participaron durante el desarrollo de este proyecto y que sin ellos no hubiera sido posible su desarrollo gracias a sus grandes contribuciones desde la óptica de sus experiencias: Alejandro Camargo (estudiante UMNG), Álvaro Gómez (estudiante UMNG), Álvaro Ruiz (docente PUJ), Andrea Caicedo (estudiante PUJ), Ángel García (docente PUJ), Fanny Sarmiento (médico especialista), Frank Cortés (estudiante UMNG), Jaime Echenique (estudiante UMNG), Jorge Echeverri Gómez (docente retirado), Jorge Pulido (estudiante UMNG), José Ignacio Valenzuela (estudiante Rosario), Juan G. Vargas (docente UMNG), Kateir Contreras (estudiante PUJ), Leonardo Maldonado (estudiante UMNG), Luisa Fernanda Campo (especialista en rehabilitación), Patricia Rodríguez (docente PUJ), Paula Rodríguez (estudiante UMNG), Pilar Garzón (estudiante UMNG) y Wilmar Peña (docente UMNG). 


\section{Referencias}

1. Forero $\mathrm{H}$. Momentos históricos de la medicina colombiana. Bogotá: Prismagraf; 2011. Enseñanza de la medicina. p. 107162 .

2. Rodríguez H. Del constructivismo al construccionismo: implicaciones educativas. Revista Educación y Desarrollo social. 2008;2(1):71-89.

3. Restrepo A. Clases magistrales versus actividades participativas en el pregrado de medicina. De la teoría a la evidencia. Revista de estudios sociales 2005; 20:83-91.

4. Otero-Ruiz E. La Investigación Médica en Colombia: Perspectivas hacia el final del Milenio. Revista Medicina. 1999;21(2):1-5.

5. Agudelo C, De la Hoz F, Mojica M, Eslava JC, Robledo R, Cifuentes $\mathrm{P}$, et al. Prioridades de investigación en salud en Colombia: perspectiva de los investigadores. Revista de salud pública. 2009;11(2):301-309.

6. Sancho, J. Docencia e investigación en la universidad: una profesión, dos mundos. Educar. 2001;28:41-60.

7. Radi, R. (2008), Esencialidad de la Investigación Biomédica en la Facultad de Medicina y su rol en el Sistema de Salud. Cuadernos del CES, Montevideo. recuperado de http://www.smu.org.uy/ elsmu/organismos/ces/cuadernos/cuadernos4/art6 el 29 de octubre 2012

8. Roselli D. La investigación biomédica en Colombia: un análisis de medline. Colombia medica. 1998;29:108-111.

9. Rojas V. Las publicaciones en revistas indexadas, único indicador de la producción de las sociedades científicas estudiantiles. CIMEL. 2007; 12: 5-6.

10. Aslam F, Shakir M, Qayum MA. Why medical students are crucial to the future of research in South Asia. Medicine. 2005;2 (11):1110-1111.

11. Liu M, Mallon WT. Tenure in transition: trends in basic science faculty appointment policies at U.S. medical schools. Academic Medicine. 2004;79:205-13.

12. Ángel AM, Botero HF, González DC, Ospina LP, Velasco MM Ocampo MF. Interés de los estudiantes de medicina por la investigación. CIMEL. 2010;15(1):9-13.

13. Hib, J. Aprendizaje basado en problemas. Facultad de medicina. Documentos. Montevideo. Recuperado de http:// www.claeh.edu.uy/medicina/medicina/doc/doc_aprendizaje_ basado_problemas.pdf el 9 de marzo de 2013.

14. Galindo L. Arango M, Díaz D, Villegas E, Aguirre C, Kambuourova M, Jaramillo P. Como el aprendizaje basado en problemas (ABP) transforma los sentidos educativos del programa de Medicina de la Universidad de Antioquia?. Iatreia. 2010;24(3):325-334

15. Finucane PM, Johnson SM, Prideaux DJ. Problem based learning: its rationale and efficacy. The Medical Journal of Australia. 1998;168:445-8

16. Sandoval HF. Aplicación del aprendizaje basado en problemas en estudiantes de medicina de la asignatura medicina interna I de la universidad nacional de Colombia sede Bogotá.. Recuperado de http://www.bdigital.unal.edu.co/7162/1/598100.2011.pdf el 9 de marzo de 2013

17. Rodríguez HM, Lugo L, Aguirre C. Aprendizaje basado en problemas, en el currículo de la facultad de medicina de la universidad de Antioquia. Iatreia. 2004;17(3):245-257.

18. Burón J. Enseñar a aprender: Introducción a la metacognición. 6ta ed. Bilbao: Ediciones Mensajero. 2002. 145p.

19. Flavell JH. Metacognition and cognitive monitoring: A new area of cognitive- developmental inquiry. American Psychologist. 1979;34:90-911.

20. Peters M. Does Constructivist Epistemology Have a Place in Nurse Education?. Journal of Nursing Education. 2000;39(4):166-170.

21. Rivers WP. Autonomy at all costs: An ethnography of metacognitive self- assessment and self-management among experienced language learners. Modern Language Journal. 2001; 85:279-290.

22. Pressley M, Wood E, Woloshyn W. Interactions Among aptitues, strategies and knowledge in cognitive performance. New York. Springer. 1990. Chapter 15, Elaborative interrogation and facilitation of fact learning: Why having a knowledge base is one thing and using it is quite another; p. 200-221.

23. Otake C. Las experiencias metacognitivas, sus estrategias y su relación con las plataformas educativas. Memorias del 6 Encuentro Nacional e Internacional de Centros de Autoacceso de Lenguas. La autonomía del aprendiente: escenarios posibles. 2006. En red México: CELE, UNAM. Recuperado de http://cad.cele.unam.mx: 8080/RD3/prueba/pdf/otake7.pdf el 1 de Marzo de 2013

24. Klimenko, O. \& Álvares, J.L. Aprender cómo aprendo: la enseñanza de estrategias metacognitivas. Educación y Educadores. 2009;12(2):11-28.

25. Vélez, I. Dávila, R. De la investigación universitaria en Colombia. Educación Superior y Desarrollo. 1984;3(1):48-54

26. Pozo J, Scheuer N, Pérez M, Mateos M, Martin E, Cruz M. Nuevas formas de pensar la enseñanza y el aprendizaje. Barcelona: Editorial Grao; 2006. La nueva cultura del aprendizaje en la sociedad del conocimiento. p. 29-54.

27. Villareal LJ. Reflexiones acerca de la escritura y los cursos de redacción. Thesaurus 1993;48(2):416-426.

28. Mayer R. Cognitive, metacognitive and motivational aspects of problem solving. Instructional Science. 1998;26:49-63. 